\title{
Efectos inducidos por Ruta graveolens L., Cnidoscolus chayamansa McVaugh y Citrus aurantium L. sobre los niveles de glucosa, colesterol y triacilglicéridos en un modelo de rata diabética
}

\author{
Lauro Figueroa-Valverde, ${ }^{* 1}$ Francisco Díaz-Cedillo, ${ }^{2}$ Abelardo Camacho-Luis, ${ }^{3}$ \\ Maria López Ramos ${ }^{1}$
}

\begin{abstract}
${ }^{1}$ Laboratorio de Investigación de la Facultad de Ciencias Químico-Biológicas, Universidad Autónoma de Campeche, Av. Agustín Melgar, Col Buenavista C.P. 24039 Campeche Cam., México

${ }^{2}$ Laboratorio de Química Orgánica de la Esc. Nal. de Ciencias Biológicas del Instituto Politécnico Nacional. Prol. Carpio y Plan de Ayala s/n, Col. D.F. C.P. 11340 Santo Tomas, México

${ }^{3}$ Facultad de Medicina de la Universidad Autónoma del Estado de Durango, Dgo., México
\end{abstract}

\begin{abstract}
RESUMO: "Efeitos induzidos por Ruta graveolens L., Rutaceae, Cnidoscolus chayamansa McVaugh, Euphorbiaceae, e Citrus aurantium L., Rutaceae, nos níveis de glucose, colesterol e triacilglicerídeos num modelo do rato diabético". Diversas plantas com propriedades hipoglicêmicas foram usadas na medicina popular e em sistemas tradicionais de curas em torno do mundo. A finalidade deste trabalho foi avaliar os efeitos induzidos por Ruta graveolens L, Rutaceae, Cnidoscolus chayamansa McVaugh, Euphorbiaceae, e Citrus aurantium L., Rutaceae, em modelo do rato diabético onde níveis da glucose foram determinados a cada $24 \mathrm{~h}$ em um mês antes da administração gástrica do extrato das plantas. Colesterol e triacilglicerídeos foram avaliados usando técnicas enzimáticas. Os resultados mostraram que a administração de Cnidoscolus chayamansa a dose de 0,5 a $1,5 \mathrm{~g} / \mathrm{kg}$ induz um aumento hipoglicêmico $(<200 \mathrm{mg} / \mathrm{dL})$. Outros dados indicam que Cnidoscolus chayamansa exerce variações nos níveis de triacilglicerídeos (80$90 \mathrm{mg} / \mathrm{dL}$ ) e colesterol (88-96 mg/dL). A administração de Citrus aurantium nas mesmas doses não foi suficiente para diminuir os níveis de glucose $(>200 \mathrm{mg} / \mathrm{dL})$. Outros resultados, mostraram que Citrus aurantium exerce mudanças na concentração de triacilglicerídeos (158-172 mg/dL) e colesterol (120-128 mg/dL). Finalmente, a administração de Ruta graveolens na dose de $0.5 \mathrm{~g} /$ $\mathrm{kg}$ induziu um efeito hipoglicêmico $(<200 \mathrm{mg} / \mathrm{dL})$. Ruta graveolens, na dose de 0.5 a $1.5 \mathrm{~g} / \mathrm{kg}$, induziu variações nos níveis de triacilglicerídeos (110-120 mg/dL) e colesterol (116-124 mg/dL). Em conclusão, a administração de Cnidoscolus chayamansa exerce efeitos hipoglicêmicos numa maneira dose dependente em comparação com Ruta graveolens e Citrus aurantium. As plantas avaliadas induzem mudanças nos níveis de lipídeos dependente da dose.
\end{abstract}

Unitermos: Diabetes, Cnidoscolus chayamansa, Ruta graveolens, Citrus aurantium, Wistar.

\begin{abstract}
Induced effects by Ruta graveolens L., Rutaceae, Cnidoscolus chayamansa McVaugh, Euphorbiaceae, and Citrus aurantium L., Rutaceae, on glucose, cholesterol and triacylglycerides levels in a diabetic rat model". Several plants with hypoglycemic properties have been used in folk medicine and traditional healing systems around the world. The purpose of this work was to evaluate the effects of Ruta graveolens L., Rutaceae, Cnidoscolus chayamansa McVaugh, Euphorbiaceae, and Citrus aurantium L., Rutaceae, in a diabetic rat model to which the glucose levels were quantified every 24 hours by one month before of gastric administration of plants extract. Additionally, the cholesterol and triacylglycerides were evaluated using standard enzymatic techniques. The results showed that increases in the dose $(0.5$ to $1.5 \mathrm{~g} / \mathrm{kg})$ of Cnidoscolus chayamansa induce a high hypoglycemic effect $(<200 \mathrm{mg} / \mathrm{dL})$. Another data indicate that Cnidoscolus chayamansa exerts variations in triacylglycerides $(80-90 \mathrm{mg} / \mathrm{dL})$ and cholesterol (88$96 \mathrm{mg} / \mathrm{dL}$ ). Nevertheless, the administration of Citrus aurantium in the same doses was not sufficient for diminish the glucose levels ( $>200 \mathrm{mg} / \mathrm{dL}$ ). Other results, showed that Citrus aurantium exert changes in the concentration of triacylglycerides (158-172 mg/dL) and cholesterol (120-128 mg/ dL). Finally, the administration of Ruta graveolens at dose of $0.5 \mathrm{mg} / \mathrm{kg}$ induces a hypoglycemic effect $(<200 \mathrm{mg} / \mathrm{dL})$. Additionally, Ruta graveolens at dose of 0.5 to $1.5 \mathrm{~g} / \mathrm{kg}$ induce variations in the triacylglycerides $(110-120 \mathrm{mg} / \mathrm{dL})$ and cholesterol $(116-124 \mathrm{mg} / \mathrm{dL})$ levels. In conclusion the administration of Cnidoscolus chayamansa it exerts hypoglycemic effects in a manner dosedependent in comparison with both Ruta graveolens and Citrus aurantium. In addition, the plants evaluated induce changes in lipids levels dose-dependent.
\end{abstract}


Keywords: Diabetes, Cnidoscolus chayamansa, Ruta graveolens, Citrus aurantium, Wistar.

\begin{abstract}
RESUMEN: Varias plantas con propiedades hipoglucemicas se han utilizado en medicina popular y sistemas curativos tradicionales en todo el mundo. El propósito de este trabajo fue evaluar los efectos inducidos por Ruta graveolens L., Rutaceae, Cnidoscolus chayamansa McVaugh, Euphorbiaceae, y Citrus aurantium L., Rutaceae, en un modelo de rata diabética, a la que se le cuantificaron los niveles de glucosa cada 24 horas por un mes después de la administración gástrica del extracto de las plantas. Además, el colesterol y los triglicéridos fueron evaluados usando técnicas enzimáticas. Los resultados mostraron que la administración de Cnidoscolus chayamansa a dosis de 0.5 a $1.5 \mathrm{~g} / \mathrm{kg}$ induce un aumento hipoglucemico $(<200 \mathrm{mg} / \mathrm{dL})$. Otros datos indican que Cnidoscolus chayamansa ejerce variaciones en los niveles de triacilglicéridos (80-90 mg/dL) y colesterol (88-96 mg/dL). Sin embargo, la administración de Citrus aurantium en las mismas dosis no fue suficiente para disminuir los niveles de glucosa (>200 mg/dL). Otros resultados, mostraron que Citrus aurantium ejerce cambios en la concentración de triacilglicéridos (158-172 mg/dL) y colesterol (120-128 mg/dL). Finalmente, la administración de Ruta graveolens a dosis de $0.5 \mathrm{~g} / \mathrm{kg}$ induce un efecto hipoglucemico $(<200 \mathrm{mg} / \mathrm{dL})$. Además, Ruta graveolens a dosis de $0.5 \mathrm{a} 1.5 \mathrm{~g} / \mathrm{kg}$ induce variaciones en los niveles de triacilglicéridos $(110-120 \mathrm{mg} / \mathrm{dL})$ y colesterol $(116-124 \mathrm{mg} /$ dL). En conclusión la administración de Cnidoscolus chayamansa ejerce efectos hipoglucemicos en una manera dosis dependiente en comparación con Ruta graveolens y Citrus aurantium. Además, las plantas evaluadas inducen cambios en los niveles de lípidos dependiente de la dosis.
\end{abstract}

Palabras clave: Diabetes, Cnidoscolus chayamansa, Ruta graveolens, Citrus aurantium, Wistar.

\section{INTRODUCCIÓN}

Uno de los problemas que actualmente enfrenta la población mundial son las enfermedades cardiovasculares (Stamler et al., 1993; Srinath et al., 1998; Gaziano, 2005). Existen varios factores de riesgo entre los que se encuentran; el tabaquismo (Ezzati et al., 2005), el alcoholismo (Dyer et al., 1977) y altas concentraciones de lípidos (Gordon et al., 1989) entre otros, que incrementan la progresión de estos desordenes cardiovasculares. Además, es importante mencionar que existen estudios epidemiológicos (Baynes, 1991; Harris et al., 1998) y clínicos (Rubin et al., 1994) donde se ha demostrado que la diabetes puede ser un factor condicionante para el desarrollo de enfermedades cardiovasculares. Aquí cabe mencionar que la diabetes a su vez esta condicionada por incrementos en los niveles de glucosa sanguínea, es así que desde hace varias décadas se han desarrollado una infinidad de medicamentos para el control de la glucosa (Oliveira et al., 1998; Moller, 2001). En este sentido, la hiperglicemia puede ser tratada inicialmente con fármacos hipoglucemiantes como son metformina (Knowler et al., 2002) y glibenclamida (O'Sullivan \& Cashman, 1970) entre otros (Zarate y Tene, 1999). Sin embargo, algunos de estos medicamentos tienden a producir algunos efectos secundarios (Wongpaitoon et al., 1981; Salper et al., 2003) y además son relativamente costosos para países en vías de desarrollo. Por lo tanto, se requiere de alternativas farmacológicas que ayuden al tratamiento de la diabetes, en este contexto, desde hace varias décadas se ha usado la medicina tradicional como una alternativa, que involucra el manejo de plantas con finalidades terapéuticas en los países que se encuentran en vías de desarrollo (Mahair \& Gulliford, 1997; Funke et al., 2006). Es así, que se ha considerado que los fitofármacos derivados de las plantas poseen menor toxicidad que algunos fármacos sintéticos (Rates, 2001). En este sentido, existen reportes sobre el manejo de medicina tradicional para el control de la diabetes (Pérez et al., 1998; Andrade \& Wiedenfield, 2001), ejemplo de esto es el uso de Cnidoscolus chayamansa por una población del sur de Texas (Hitchcock et al., 1997) y en regiones mexicanas (Coronado et al., 2004) para el control de diabetes mellitus. En otros, estudios se han realizado experimentos en un modelo de rata diabética los cuales señalan que la administración subcronica de Cnidoscolus chayamansa disminuye los niveles de glucosa y lípidos (Palos , 2007).

Por otro lado, existen datos reportados por Sugiura y colaboradores (2006) quienes señalan que la administración del extracto de Citrus (unshiu), ejerce variación en los niveles de glucosa en un modelo de ratas (GK) diabéticas tipo 2. Aunado a esto, otros reportes realizados por Singh y Kar indican que la administración oral de Citrus sinensis $(25 \mathrm{mg} / \mathrm{kg})$ en ratones diabéticos induce disminución en los niveles de glucosa (Singh et al., 2007). Sin embargo otros estudios (Colker et al., 1999) muestran que la administración de $975 \mathrm{mg}$ Citrus auriantum en jóvenes sanos de 21 años no afectaron los niveles de glucosa $(89 \mathrm{mg} / \mathrm{dL})$ en comparación con los varones de la misma edad usados como control $(88 \mathrm{mg} / \mathrm{dL})$. Por otro lado, otros estudios indican que algunas sustancias (Je et al., 2002) envueltas en otro tipo de plantas como es el caso de Ruta graveolens tambien pueden afectar los niveles de glucosa, sin embargo, es importante mencionar que existen reportes donde se señala que la administración de Ruta graveolens puede inducir efectos tóxicos (Agra et al., 2002).

Por lo anteriormente descrito, los estudios 
enfocados a analizar los efectos que inducen Cnidoscolus chayamansa, Ruta graveolens y Citrus aurantium sobre los niveles de glucosa y la presión sanguínea, no solamente son escasos sino que han arrojado hasta la fecha resultados que son frecuentemente difíciles de interpretar y a menudo contradictorios. Parte de la dificultad en la interpretación puede ser el abordaje que cada investigador realiza ó por las diferentes dosis administradas. Por lo tanto, el presente trabajo tuvo el propósito de evaluar el efecto que ejerce Cnidoscolus chayamansa, Ruta graveolens y Citrus aurantium sobre los niveles de glucosa, peso, colesterol y triacilglicéridos (in vivo) en un modelo de rata diabética.

\section{MATERIAL Y MÉTODOS}

\section{Material vegetal}

La Ruta graveolens, la Cnidoscolus chayamansa y el Citrus aurantium fueron adquiridos en el mercado principal de la $\mathrm{Cd}$. de Campeche proveniente de los municipios adyacentes. El material vegetal fue autentificado por personal académico de la Universidad Autónoma de Campeche con número de registro: Ruta graveolens (UCAM-4445), Cnidoscolus chayamansa (UCAM-4319), y Citrus aurantium (UCAM-10870).

\section{Animales}

Todos los protocolos fueron aprobados por el comité de ética de la Universidad Autónoma de Campeche, su aprobación se basó en la guía para cuidados y uso de animales de laboratorio, publicado por la Academia Nacional de Ciencias, Washington D.C., (Bayne, 1996). La cepa que se utilizo fue ratas machos Wisstar (220-250 g). Antes de su experimentación se mantuvieron a una temperatura de $22{ }^{\circ} \mathrm{C}$ y ciclo claro/oscuro de $12 \mathrm{~h}$. Los animales fueron alimentados con una dieta balanceada (purina) y agua para mantenerlas en condiciones deseables.

\section{Extractos}

\section{Metodo general}

El extracto de las plantas de estudio (Ruta graveolens, la Cnidoscolus chayamansa y Citrus aurantium) fue preparado por medio de una extracción continua usando el aparto soxhlet (Hawthorne et al., 2000). La extracción de una muestra de $20 \mathrm{~g}$ de hojas previamente secas se realizo usando como disolvente $250 \mathrm{~mL}$ metanol $(80 \%)$ por espacio de 8 h. Después del tiempo de extracción el volumen de la solución alcohólica se redujo a presión reducida en un rotovapor (Buchi B-490, Labortechnick, Zwitzerland). Al producto obtenido se le adiciono una mezcla de disolventes cloroformo:agua (v/v, 4:1) para separar la fase orgánica de la acuosa. El volumen de la fase orgánica se redujo hasta sequedad a presión reducida y la mezcla obtenida se restituyo con etanol (70\%) para usarla como solución madre en este estudio.

\section{Identificación de flavonoides}

El análisis espectroscópico de los flavonoides involucrados en Ruta graveolens, Cnidoscolus chayamansa y Citrus aurantium fue realizado a una longitud de onda en un rango de 200 a $500 \mathrm{~nm}$ como lo señala Heimler et al. (1992). Es importante mencionar que se realizo una curva patrón utilizando dihydromyricetina y amentoflavona rutina, quercetina, naringina, hesperidina y nobiletina.

\section{Inducción de diabetes}

Las ratas fueron inyectadas con alloxan monohidratado disuelto en solución salina a una dosis de $150 \mathrm{mg} / \mathrm{kg}$ de peso corporal vía intraperitoneal como lo describe Sharma et al. (2008). A partir de la primera semana después de la inyección, se monitorearon los niveles de glucosa y se estableció híperglicemia cuando la concentración de glucosa fue $\geq 200 \mathrm{mg}$.

\section{Determinación del peso}

La determinación del peso corporal de los animales de estudio fue evaluado antes y después de cada tratamiento (cada $24 \mathrm{~h}$ ) por 30 días.

\section{Determinación de glucosa en forma aguda in vivo}

Se evaluaron los niveles de glucosa antes y después de cada tratamiento (cada 24 h) por 30 días. La muestra sanguínea fue colectada del extremo de la cola de cada una de las ratas y puesta en las tiras reactivas para su análisis con el glucómetro Accu-Chek, Roche company U.S.A (Hawkins, 2005).

Administración de extractos
hipoglucemiantes

Las ratas fueron divididas en varios grupos después de la inducción de la diabetes (Tabla 1) a las cuales se les administro el extracto de cada una de las plantas involucradas en este trabajo como son; Ruta graveolens, Cnidoscolus chayamansa y Citrus aurantium en dosis de $0.5 \mathrm{mg} / \mathrm{kg}$ a $1.5 \mathrm{mg} / \mathrm{kg}$ usando un tubo intragastríco por 30 días. Las diferentes dosis administradas fueron disueltas en $1 \mathrm{~mL}$ de agua, misma que fue administrada a cada uno de los animales de estudio. Además se administraron Glibenclamida en una dosis de $600 \mu \mathrm{g} / \mathrm{kg}$ (Rajasekaran et al., 2005) y dosis de $350 \mathrm{mg} / \mathrm{kg}$ de metformina (Penicaud et al., 1989) para ser usados como controles. Finalmente, se usaron como controles ratas sin diabetes/sin tratamiento y ratas con diabetes/sin tratamiento a las cuales solo se les 
administro solución salina $(1 \mathrm{~mL})$.

Determinación de glucosa, colesterol y triacilglicéridos en forma crónica

Para evaluar estos parámetros se sacrificaron los animales y se extrajo la muestra sanguínea $(2 \mathrm{~mL})$ y se deposito en tubos que contenían $0.46 \mathrm{~mL}$ de EDTA (ácido etilendiaminotetraacético) para el análisis especifico de cada uno de los parámetros de estudio.

\section{Glucosa}

Los niveles de glucosa fueron cuantificados por el método de glucosa oxidasa-peroxidasa. Se adiciono 1 $\mathrm{mL}$ del reactivo de trabajo (Reactivo 4-aminofenazona/ enzi-mas + Reactivo Fenol [solución de fenol $5 \mathrm{mmol} / \mathrm{L}$ en buffer de fosfato $100 \mathrm{mmol} / \mathrm{L}, \mathrm{pH}=7.0]$ ). La solución obtenida se mezcló hasta homogenizar y se incubó durante $10 \mathrm{~min}$ a $37^{\circ} \mathrm{C}$. Aquí es importante mencionar que la glucosa se determina por oxidación enzimática en presencia de glucosa oxidasa y el peroxido de hidrógeno formado, en presencia de peroxidada, oxida el cromógeno 4-aminofenazona/fenol. Los niveles de glucosa fueron cuantificados mediante lecturas de absorbancia en el espectrofotómetro a $520 \mathrm{~nm}$ (Lott \& Turner, 1975).

\section{Triacilglicéridos}

La concentración de triacilglicéridos se obtuvo mediante la técnica glicerol fosfato oxidasa/peroxidada (Fossati \& Prencipe, 1982). A $10 \mu \mathrm{L}$ de suero o plasma se le adicionó $1 \mathrm{~mL}$ del reactivo de trabajo que está compuesto de; pipes (acido 1,4-piperazina-dietano-sulfonico, 45 $\mathrm{mmol} / \mathrm{L}), p$-clorofenol $(6 \mathrm{mmol} / \mathrm{L})$, cloruro de manganeso ( $5 \mathrm{mmol} / \mathrm{L})$, lipasa $>100 \mathrm{U} / \mathrm{mL}$, glicerol quinasa $>1.5 \mathrm{U} /$ $\mathrm{mL}$, glicerol-3-fosfato oxidasa $>4 \mathrm{U} / \mathrm{mL}$, peroxidasa $>0.8$ $\mathrm{U} / \mathrm{mL}$, 4-aminoantipirina $0.75 \mathrm{mmol}$, ATP $0.9 \mathrm{mmol}$, $\mathrm{pH}$ 7.0. La solución obtenida se mezcló hasta homogenizar y se incubó durante $5 \mathrm{~min}$ a $37^{\circ} \mathrm{C}$. El registro de la absorvancia de la muestra se realizó a $500 \mathrm{~nm}$ y la concentración de triacilglicéridos se calculó por el método reportado por Bucolo (1973).

\section{Colesterol}

Los niveles de colesterol fueron cuantificados enzimáticamente usando la técnica de colesterol oxidasa/ peroxidada (Allain et al., 1974). Se adicionó $1 \mathrm{~mL}$ del reactivo de trabajo (Reactivo 4-aminofenazona/Enzimas + Reactivo Fenol [solución de fenol $5 \mathrm{mmol} / \mathrm{L}$ en buffer de fosfato $50 \mathrm{mmol} / \mathrm{L}, \mathrm{pH}=7.0]$ ) a $10 \mu \mathrm{L}$ de muestra (plasma) y se agitó hasta obtener una mezcla homogénea. La solución obtenida se incubó a $37{ }^{\circ} \mathrm{C}$ durante $15 \mathrm{~min}$ a temperatura ambiente. La cuantificación de los niveles de colesterol se llevo a cabo mediante lecturas de absorvancia, aquí es importante mencionar que la determinación de colesterol se basa en la conversión de colesterol a colest-4en-3-ona, este compuesto tiene un espectro de absorción a $240 \mathrm{~nm}$.

\section{Análisis estadístico}

Todos los análisis se desarrollaron usando el Software SPSS 12.0. Los valores obtenidos fueron expresados como la media \pm error estándar de la media. Las diferencias se consideraron significativas cuando $p$ tiene un valor igual o menor a 0.05 .

\section{RESULTADOS}

\section{Identificación de flavonoides mediante espectrofotometría de ultravioleta}

Los resultados del análisis de espectrofotometría de ultravioleta de los flavonoides evaluados en este estudio (Tabla 2) muestran variaciones en la longitud de onda para cada flavonoide en un rango de 240 a $380 \mathrm{~nm}$.

\section{Determinación del peso}

Los resultados mostrados en la Tabla 3 (en un lapso de 30 días) señalan que la administración de Cnidoscolus chayamansa y Ruta graveolens a dosis de 0.5 $\mathrm{g} / \mathrm{kg}$ mostraron diferencias significativas $(p=0.005)$ con respecto al control (ratas diabéticas sin tratamiento), pero con respecto a las ratas no diabéticas. Otros datos, señalan que la administración de Citrus auriuantum a dosis de 1.0 y $1.5 \mathrm{~g} / \mathrm{kg}$ ejercieron incrementos en el peso de las ratas en comparación con las ratas control (normal y diabéticas sin tratamiento).

\section{Cuantificación de glucosa en forma aguda}

Los datos obtenidos mostraron que los niveles de glucosa en condiciones control (ratas diabéticas sin tratamiento farmacológico) fluctuaron entre 405 a 490 $\mathrm{mg} / \mathrm{dL}$ (Figura 1) en un lapso de 30 días. Otros resultados encontrados en el grupo de ratas con tratamiento farmacológico mostraron 1) la aplicación de glibenclamida indujo una reducción significativa $(p=0.005)$ en los niveles de glucosa (490 a $110 \mathrm{mg} / \mathrm{dL}$ ) través del tiempo; 2) la administración de metformina mostró un efecto hipoglucemiante en ratas diabéticas (450 a $150 \mathrm{mg} / \mathrm{dL} ; p$ $=0.005)$. Además, otros obtenidos de la evaluación de la administración de Cnidoscolus chayamansa a dosis de 1) $0.5 \mathrm{~g} / \mathrm{kg}$ mostraron un decremento en los niveles de glucosa (456 a $198 \mathrm{mg} / \mathrm{dL}) ; 2) 1.0 \mathrm{~g} / \mathrm{kg}$ se encontró una reducción significativa $(p=0.005)$ en la concentración de glucosa (416 a $118 \mathrm{mg} / \mathrm{dL})$; y 3) 1.5 mostró una disminución significativa $(p=0.005)$ en los niveles de glucosa (420 a $102 \mathrm{mg} / \mathrm{dL})$. 
Otros resultados de experimentos alternativos mostraron que el grupo de ratas diabéticas tratadas con Ruta graveolens (Figura 2) a dosis de a) $0.5 \mathrm{~g} / \mathrm{kg}$ mostraron un decremento significativo $(p=0.005)$ en los niveles de glucosa (422 a $121 \mathrm{mg} / \mathrm{dL}) ; \mathrm{b}) 1.0 \mathrm{~g} / \mathrm{kg}$ se encontró una reducción en la concentración de glucosa en los rangos de 466 a $290 \mathrm{mg} / \mathrm{dL}$; c) $1.5 \mathrm{~g} / \mathrm{kg}$ mostraron una reducción en los niveles de glucosa ( 480 a $270 \mathrm{mg} / \mathrm{dL}$ ).

Finalmente, los niveles de glucosa fueron determinados por la administración de Citrus aurantium (Figura 4). Los resultados mostraron que a dosis de 1) 0.5 $\mathrm{g} / \mathrm{kg}$ hubo una reducción en la concentración de glucosa (458 a $266 \mathrm{mg} / \mathrm{dL}) ; 2) 1.0 \mathrm{~g} / \mathrm{kg}$ mostraron una disminución de 434 a $245 \mathrm{mg} / \mathrm{dL}$; y 3) $1.5 \mathrm{~g} / \mathrm{kg}$ se hallaron niveles de glucosa que fluctuaron entre 444 y $260 \mathrm{mg} / \mathrm{dL}$.

Tabla 1. Diseño experimental. Distribución de grupos y dosis administradas de sustancias involucradas en el estudio.

\begin{tabular}{lll}
\hline \multicolumn{1}{c}{ Grupo } & \multicolumn{1}{c}{ Substancia } & \multicolumn{1}{c}{ Dosis } \\
\hline Ratas normales (control 1) & Solución salina & $1 \mathrm{ml}$ \\
Ratas diabéticas (control 2) & Solución salina & $1 \mathrm{ml}$ \\
Ratas diabéticas (Grupo I) & Glibenclamida & $600 \mu \mathrm{g} / \mathrm{kg}$ \\
Ratas diabéticas (Grupo II) & Metformina & $350 \mathrm{mg} / \mathrm{kg}$ \\
Ratas diabéticas (Grupo III) & Cnidoscolus chayamansa & $(0.5 \mathrm{~g} / \mathrm{kg})$ \\
Ratas diabéticas (Grupo IV) & Cnidoscolus chayamansa & $(1.0 \mathrm{~g} / \mathrm{kg})$ \\
Ratas diabéticas (Grupo V) & Cnidoscolus chayamansa & $(1.5 \mathrm{~g} / \mathrm{kg})$ \\
Ratas diabéticas (Grupo VI) & Ruta graveolens & $(0.5 \mathrm{~g} / \mathrm{kg})$ \\
Ratas diabéticas (Grupo VII) & Ruta graveolens & $(1.0 \mathrm{~g} / \mathrm{kg})$ \\
Ratas diabéticas (Grupo VIII) & Ruta graveolens & $(1.5 \mathrm{~g} / \mathrm{kg})$ \\
Ratas diabéticas (Grupo IX) & Citrus aurantium & $(0.5 \mathrm{~g} / \mathrm{kg})$ \\
Ratas diabéticas (Grupo X) & Citrus aurantium & $(1.0 \mathrm{~g} / \mathrm{kg})$ \\
Ratas diabéticas (Grupo XI) & Citrus aurantium & $(1.5 \mathrm{~g} / \mathrm{kg})$ \\
\hline
\end{tabular}

\section{Cuantificación de glucosa, colesterol y triacilglicéridos en forma crónica}

\section{Determinación de glucosa}

Los resultados mostrados en la Tabla 3 señalan que la administración de glibenclamida (114 mg/dL) mostró efectos hipoglucemiantes $\approx$ similares a dosis administrada de $1.5 \mathrm{~g} / \mathrm{kg}$ de Cnidoscolus chayamansa $(105 \mathrm{mg} / \mathrm{dL})$ y a la dosis de $0.5 \mathrm{~g} / \mathrm{kg}$ de Ruta graveolens $(118 \mathrm{mg} / \mathrm{dL})$. Sin embargo, la aplicación de Citrus aurantium mostró variaciones en las diferentes dosis $(238-259 \mathrm{mg} / \mathrm{dL})$.

\section{Cuantificación de triacilglicéridos}

En la Tabla 4 se muestran los niveles de triacilglicéridos encontrados por la administración de Cnidoscolus chayamansa señalan una variación de 80 a $90 \mathrm{mg} / \mathrm{dL}$ en las tres diferentes dosis. Otros datos indican que la aplicación de Ruta graveolens a las diferentes dosis fluctuaron entre 110 a $120 \mathrm{mg} / \mathrm{dL}$. Finalmente, otros

Tabla 2. Análisis espectrofotométrico de ultravioleta. Espectros encontrados de los diferentes flavonoides presentes en Cnidoscolus chayamansa ${ }^{1}$, Ruta graveolens ${ }^{2}$ y Citrus aurantium ${ }^{3}$ en un rango de 200-400 $\mathrm{nm}$.

\begin{tabular}{lc}
\hline Flavonoide & $\boldsymbol{\lambda}_{\max }(\boldsymbol{\varepsilon})$ \\
\hline Dihidromiricetina $^{l}$ & 242 \\
Amentoflavona $^{l}$ & 330 \\
Rutina $^{2}$ & 362 \\
Quercetina $^{2}$ & 380 \\
Naringina $^{3}$ & 372 \\
Hesperidina $^{3}$ & 286 \\
Nobiletina $^{3}$ & 334
\end{tabular}

Tabla 3. Determinación de los niveles del peso y glucosa por la administración de Cnidoscolus chayamansa, Ruta graveolens, Citrus aurantium y los controles (glibenclamida y metformina) en ratas diabéticas en forma crónica.

\begin{tabular}{lcc}
\hline Grupos & peso $(\mathrm{g}){ }^{*}$ & Glucosa $(\mathrm{mg} / \mathrm{dL})^{*}$ \\
\hline Normal & $240 \pm 3.05$ & $80 \pm 8.06$ \\
Diabéticas (control) & $190 \pm 4.64$ & $444 \pm 3.30$ \\
Diabéticas + Glibenclamida $(600 \mu \mathrm{g} / \mathrm{kg})$ & $259 \pm 8.28$ & $114 \pm 6.10$ \\
Diabéticas + Metformina $(350 \mathrm{mg} / \mathrm{kg})$ & $248 \pm 6.34$ & $146 \pm 10.17$ \\
Diabéticas + Chidoscolus chayamansa $(0.5 \mathrm{~g} / \mathrm{kg})$ & $232 \pm 7.78$ & $190 \pm 6.18$ \\
Diabéticas + Chidoscolus chayamansa $(1.0 \mathrm{~g} / \mathrm{kg})$ & $242 \pm 5.08$ & $122 \pm 8.44$ \\
Diabéticas + Cnidoscolus chayamansa $(1.5 \mathrm{~g} / \mathrm{kg})$ & $244 \pm 6.34$ & $105 \pm 8.04$ \\
Diabéticas + Ruta graveolens $(0.5 \mathrm{~g} / \mathrm{kg})$ & $240 \pm 9.02$ & $118 \pm 10.02$ \\
Diabéticas + Ruta graveolens $(1.0 \mathrm{~g} / \mathrm{kg})$ & $248 \pm 6.18$ & $284 \pm 8.80$ \\
Diabéticas + Ruta graveolens $(1.5 \mathrm{~g} / \mathrm{kg})$ & $230 \pm 8.04$ & $276 \pm 7.78$ \\
Diabéticas + Citrus aurantium $(0.5 \mathrm{~g} / \mathrm{kg})$ & $230 \pm 6.04$ & $259 \pm 8.04$ \\
Diabéticas + Citrus aurantium $(1.0 \mathrm{~g} / \mathrm{kg})$ & $308 \pm 7.08$ & $238 \pm 10.02$ \\
Diabéticas + Citrus aurantium $(1.5 \mathrm{~g} / \mathrm{kg})$ & $358 \pm 5.46$ & $252 \pm 8.24$ \\
\hline
\end{tabular}

*Los valores representan la media \pm error estándar de la media. 
Tabla 4. Datos obtenidos por la administración de Cnidoscolus chayamansa, Ruta graveolens, Citrus aurantium y los controles (glibenclamida y metformina) sobre los niveles de triglicéridos y colesterol en forma crónica en ratas diabéticas en forma crónica.

\begin{tabular}{lcc}
\hline Grupos & peso $(\mathrm{g}){ }^{*}$ & Glucosa $(\mathrm{mg} / \mathrm{dL})^{*}$ \\
\hline Normal & $98 \pm 7.74$ & $78 \pm 6.18$ \\
Diabéticas (control) & $118 \pm 6.64$ & $106 \pm 8.02$ \\
Diabéticas + Glibenclamida $(600 \mu \mathrm{g} / \mathrm{kg})$ & $92 \pm 8.04$ & $118 \pm 10.02$ \\
Diabéticas + Metformina $(350 \mathrm{mg} / \mathrm{kg})$ & $79 \pm 6.04$ & $130 \pm 6.20$ \\
Diabéticas + Cnidoscolus chayamansa $(0.5 \mathrm{~g} / \mathrm{kg})$ & $96 \pm 6.68$ & $80 \pm 8.84$ \\
Diabéticas + Cnidoscolus chayamansa $(1.0 \mathrm{~g} / \mathrm{kg})$ & $90 \pm 10.12$ & $90 \pm 8.02$ \\
Diabéticas + Cnidoscolus chayamansa $(1.5 \mathrm{~g} / \mathrm{kg})$ & $88 \pm 7.02$ & $88 \pm 10.04$ \\
Diabéticas + Ruta graveolens $(0.5 \mathrm{~g} / \mathrm{kg})$ & $116 \pm 6.64$ & $120 \pm 7.76$ \\
Diabéticas + Ruta graveolens $(1.0 \mathrm{~g} / \mathrm{kg})$ & $124 \pm 8.46$ & $110 \pm 6.64$ \\
Diabéticas + Ruta graveolens $(1.5 \mathrm{~g} / \mathrm{kg})$ & $120 \pm 9.02$ & $112 \pm 6.68$ \\
Diabéticas + Citrus aurantium $(0.5 \mathrm{~g} / \mathrm{kg})$ & $128 \pm 6.64$ & $158 \pm 8.24$ \\
Diabéticas + Citrus aurantium $(1.0 \mathrm{~g} / \mathrm{kg})$ & $122 \pm 10.02$ & $164 \pm 6.34$ \\
Diabéticas + Citrus aurantium $(1.5 \mathrm{~g} / \mathrm{kg})$ & $120 \pm 8.34$ & $172 \pm 8.02$ \\
\hline
\end{tabular}

*Los valores representan la media \pm error estándar de la media.

CONTROL

CNIDOSCOLUS CHAYAMANSA $(0.5 \mathrm{~g} / \mathrm{kg})$

$\nabla$ CNIDOSCOLUS CHAYAMANSA $(1.0 \mathrm{~g} / \mathrm{kg})$

$\nabla$ CNIDOSCOLUS CHAYAMANSA $(1.5 \mathrm{~g} / \mathrm{kg})$

- METFORMINA

$\square \quad$ GLIBENCLAMIDA

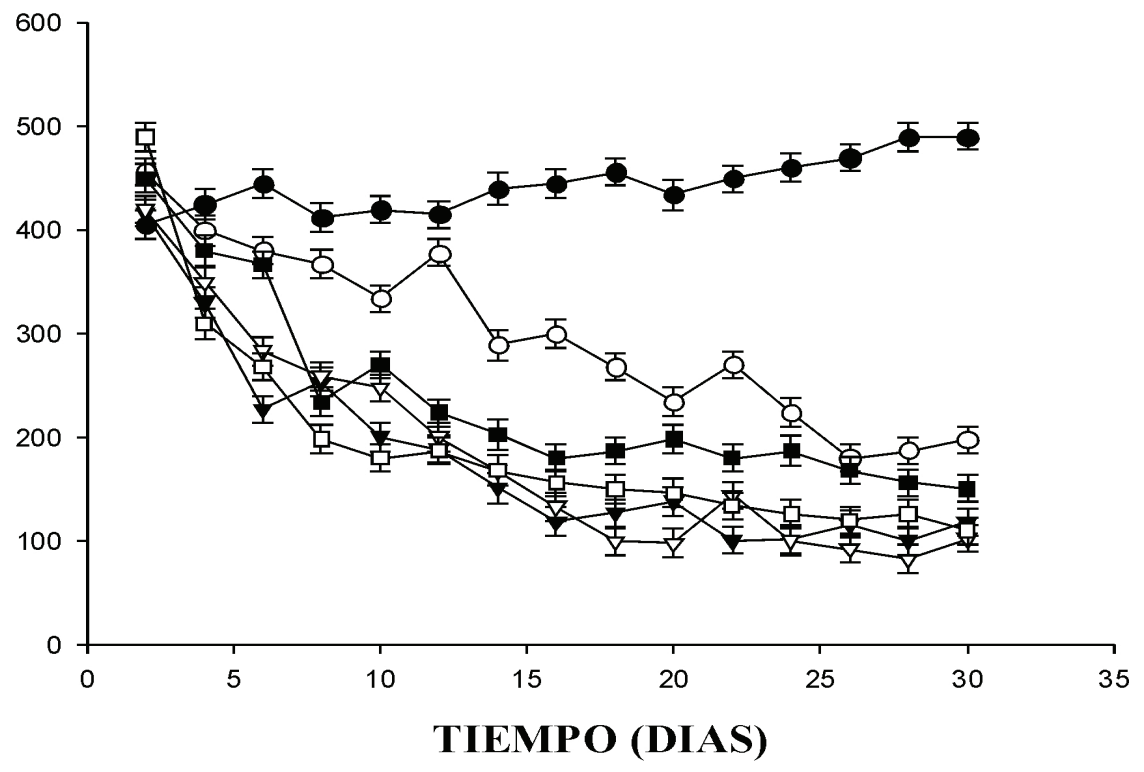

Figura 1. Efectos hipoglucemiantes de Cnidoscolus chayamansa. En la gráfica se observa que la glibenclamida $(600 \mu \mathrm{g} / \mathrm{kg})$ y la metformina $(350 \mathrm{mg} / \mathrm{kg}$ ) decrementan los niveles de glucosa en comparación con las condiciones control (ratas diabeticas sin tratamiento). Otros resultados muestran que Cnidoscolus chayamansa disminuye la concentración de glucosa dependiente de la dosis $(0.5$ a $1.5 \mathrm{~g} / \mathrm{Kg})$. Los efectos se expresan como área bajo la curva y cada punto representa la media \pm error estándar de la media. Las comparaciones fueron realizadas entre grupos para el mismo período $(\boldsymbol{p} \leq 0,05)$. 
- CONTROL

RUTA GRAVEOLENS $(0.5 \mathrm{~g} / \mathrm{kg})$

$\checkmark$ RUTA GRAVEOLENS $(1.0 \mathrm{~g} / \mathrm{kg})$

$\nabla$ RUTA GRAVEOLENS $(1.5 \mathrm{~g} / \mathrm{kg})$

- METFORMINA

a GLIBENCLAMIDA

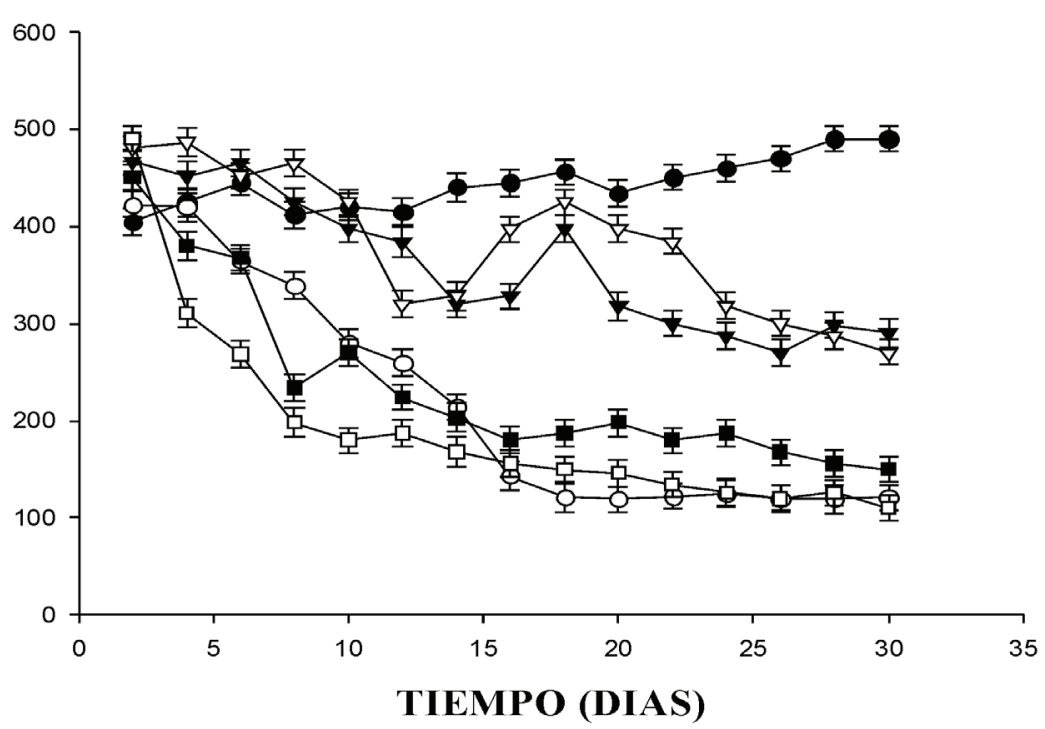

Figura 2. Actividad inducida por Ruta graveolens. En la grafica se observa el efecto inducido por glibenclamida $(600 \mu \mathrm{g} / \mathrm{kg}) \mathrm{y}$ la metformina $(350 \mathrm{mg} / \mathrm{kg}$ ) sobre los niveles de glucosa. Otros datos muestran que la Ruta graveolens disminuye los niveles de glucosa a dosis de $0.5 \mathrm{~g} / \mathrm{kg}$ en comparación con las condiciones control (ratas diabeticas sin tratamiento). Los efectos se expresan como área bajo la curva y cada punto representa la media \pm error estándar de la media. Las comparaciones fueron realizadas entre grupos para el mismo período $(\boldsymbol{p} \leq 0,05)$.

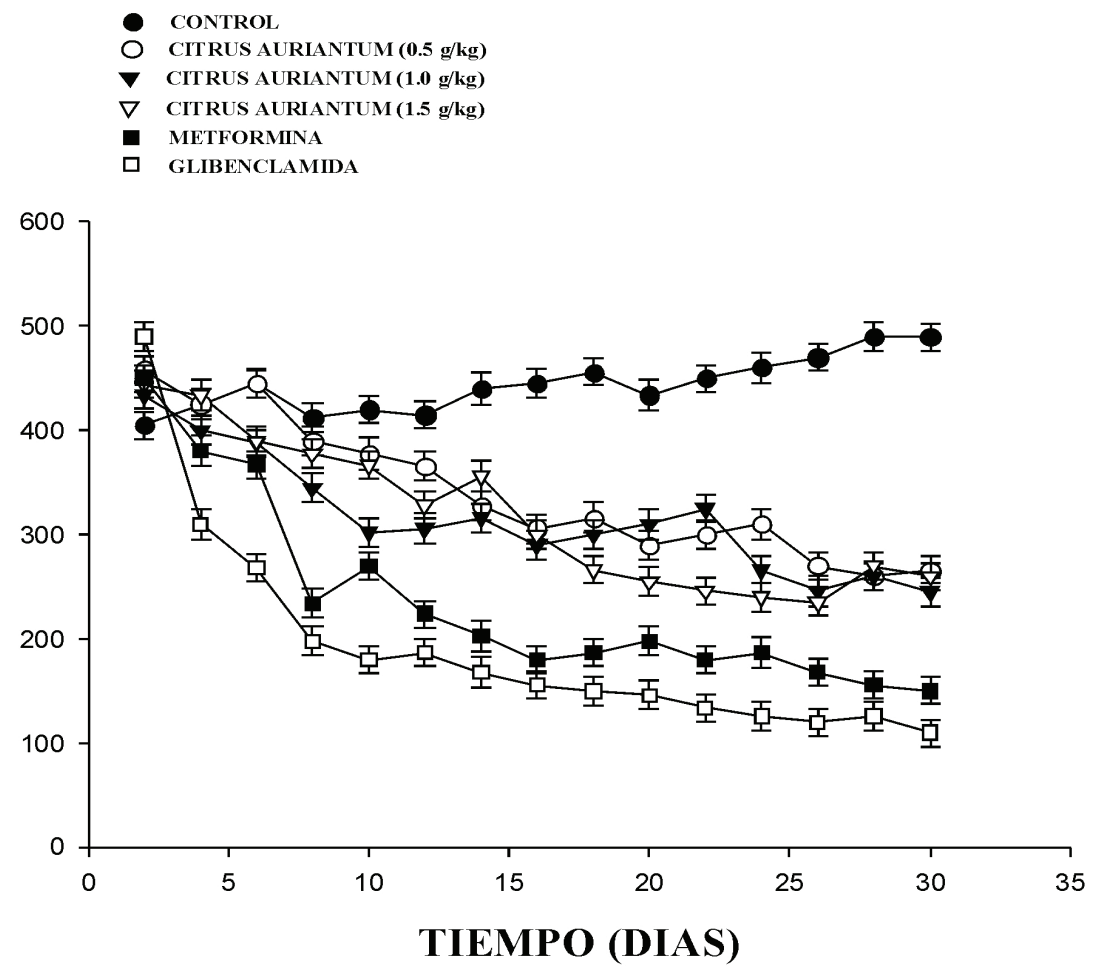

Figura 3. Efecto inducido por Citrus aurantium sobre los niveles de glucosa. Aquí se observa que la glibenclamida $(600 \mu \mathrm{g} / \mathrm{kg})$ y la metformina $(350 \mathrm{mg} / \mathrm{kg})$ disminuyen los niveles de glucosa. Otros datos señalan que Citrus aurantium induce variaciones en la concentración de glucosa en dosis de 0.5 a $1.5 \mathrm{~g} / \mathrm{kg}$ en comparación con las condiciones control (ratas diabeticas sin tratamiento). Los efectos se expresan como área bajo la curva y cada punto representa la media \pm error estándar de la media. Las comparaciones fueron realizadas entre grupos para el mismo período $(\boldsymbol{p} \leq 0,05)$. 
datos encontrados señalan que la administración de Citrus aurantium a dosis de 0.5 a $1.5 \mathrm{~g} / \mathrm{kg}$ mostraron variaciones de 158 a $172 \mathrm{mg} / \mathrm{dL}$.

\section{Evaluación de la concentración de colesterol}

Los resultados mostrados en la Tabla 4, indican que la administración de Ruta graveolens ejerció variaciones en los niveles de colesterol (116 a $124 \mathrm{mg} /$ $\mathrm{dL}$ ) dependiente de la dosis. Además, otros datos señalan que la administración de Citrus aurantium a dosis de 0.5 a $1.5 \mathrm{~g} / \mathrm{kg}$ mostraron variaciones entre 120 a $128 \mathrm{mg} / \mathrm{dL}$. Finalmente, nuestros resultados señalan que los niveles de colesterol fluctuaron entre 88 a $96 \mathrm{mg} / \mathrm{dL}$ cuando se administro Cnidoscolus chayamansa en una manera dosis dependiente.

\section{DISCUSIÓN}

En este estudio, se evalúo el efecto que inducen Cnidoscolus chayamansa, Citrus aurantium y Ruta graveolens sobre los niveles de peso, glucosa, colesterol, triacilglicéridos (in vivo) en un modelo de rata diabética. Nuestros resultados indicaron que la administración de Citrus aurantium induce incrementos en el peso de las ratas diabéticas siendo este efecto dependiente de la dosis $(1.0$ a $1.5 \mathrm{~g} / \mathrm{kg}$ ) en comparación con los efectos inducidos por Cnidoscolus chayamansa y Ruta graveolens. Es valido, mencionar que estos datos son contrarios a los reportados por algunos investigadores, los cuales señalan que algunas sustancias contenidas en Citrus aurantium pueden inducir perdida de peso corporal (Fugh-Berman \& Myers, 2004).

Por otro lado, los resultados de experimentos alternativos donde se evaluó el efecto ejercido por Cnidoscolus chayamansa sobre ratas diabéticas, mostraron que los niveles de glucosa disminuyeron significativamente por la administración de Cnidoscolus chayamansa en una manera dosis dependiente. Además, es importante mencionar que a dosis de $1.5 \mathrm{~g} / \mathrm{kg}$ el efecto hipoglucemiente inducido por Cnidoscolus chayamansa fue similar al ejercido por la glibenclamida y diferente con respecto a la actividad hipoglucemiante ejercida por metformina. Por lo tanto, este fenómeno podría estar condicionado directamente por las características químicas de las sustancias usadas como control (glibenclamida y metformina) y por las sustancias involucradas en la estructura de Cnidoscolus chayamansa. Nosotros pensamos que posiblemente el efecto hipoglucemiante inducido por Cnidoscolus chayamansa podría depender de los flavonoides presentes en el extracto administrado en las ratas diabéticas. Por lo que en este trabajo fue realizado un análisis de espectrofotometría de ultravioleta con la finalidad de caracterizar cualitativamente los tipos de flavonoides presentes en los extractos obtenidos de Cnidoscolus chayamansa. Nuestros resultados mostraron dos señales para dihydromyricetina y amentoflavona siendo similares a los reportados por otros investigadores (Heimler et al., 1992). En este sentido, es importante mencionar que se han encontrado flavonoides en las hojas de Cnidoscolus chayamansa como son dihydromyricetina y amentoflavona [3',8"-diapigenin] (González et al., 2003) que pueden ser los responsable indirectos del efecto hipoglucemienate de Cnidoscolus chayamansa. Esta premisa es soportada por los estudios realizados por Cheng et al. (2008) donde se señala que la administración intragastrica de dihydromyricetin disminuye la concentración de glucosa en un modelo de ratas de intolerancia a la glucosa. Aunado a esto, existen datos reportados por Jong et al. (2000) los cuales muestran que la amentoflavone y otras sustancias pueden tener actividad sobre la alfa-glucosidasa y consecuentemente sobre los niveles de glucosa. Por lo tanto, estos flavonoides involucrados en la estructura de Cnidoscolus chayamansa pueden condicionar indirectamente los niveles de glucosa. Además, es importante los resultados de la administración de Cnidoscolus chayamansa mostraron diferencias en los niveles de colesterol y triglicéridos con respecto a las ratas diabéticas control (sin tratamiento), sin embargo en comparación con las ratas normales (no diabéticas/sin tratamiento) no se observo diferencias en la concentración lipídica. Estos últimos datos son similares a los reportados por otros investigadores (Palos, 2007), los cuales señalan que la administración Cnidoscolus chayamansa ejerce un efecto sobre el perfil lipídico.

Por otro lado, nos pareció importante comparar el efecto inducido por Ruta graveolens con el efecto ejercido por Cnidoscolus chayamansa. Nuestros resultados indican que Ruta graveolens mostró efectos contrarios a Cnidoscolus chayamansa dependientes de la dosis. Es así, que la administración de Ruta graveolens a dosis bajas $(0.5 \mathrm{~g} / \mathrm{kg})$ mostró actividad hipoglucemiante, sin embargo cuando se incremento la dosis este efecto disminuyo significativamente. Este fenómeno podría deberse por un lado, a la presencia de diferentes tipos de flavonoides presente en Ruta graveolens como son la rutina y la quercetina (Saieed et al., 2006) en comparación con Cnidoscolus chayamansa y por otro, a la posibilidad de que al aumentar la dosis se este activando otro mecanismo por el cual los niveles de glucosa se incrementen. Por lo tanto, el efecto ejercido por Ruta graveolens al menos es inducido parcialmente a dosis bajas. Esta hipótesis, es avalada por los estudios realizados por Srinivasan et al. (2005) los cuales administraron rutina via-oral (200 mg/ $\mathrm{kg}$ ) en ratas con diabetes, sus resultados señalan que la rutina decremento parcialmente los niveles de glucosa. Otros datos, señalan que otro flavonoide involucrado en la estructura fotoquímica de la Ruta graveolens como es el caso de la quercetina tiene influencia sobre los niveles de glucosa, en este sentido, existen reportes donde se señala que la quercetina a dosis de 10 y $50 \mathrm{mg} / \mathrm{kg}$ promueve normalización en los niveles de glucosa en un modelo de ratas diabéticas (Nuraliev et al., 1992). Estos datos confirman que el efecto hipoglucemiante inducido por Ruta 
graveolens puede involucrar este tipo de flavonoides.

Por otro lado, otros resultados señalan que la administración de Ruta graveolens no mostró diferencias en los niveles de colesterol con respecto a las ratas diabéticas control (sin tratamiento). Sin embargo, se observo un decremento en la concentración de triglicéridos de manera dosis-dependiente. Estos datos son parecidos a los reportados por Santos et al. (1999) quienes señalan que la administración de rutina indujo reducción en los niveles de triglicéridos. Aquí es importante señalar que pensando en la diferencias que encontramos sobre la actividad hipoglucemiante entre Ruta graveolens y Cnidoscolus chayamansa, en este trabajo se realizaron experimentos alternativos, donde se administro vía-oral Citrus aurantium a las mismas dosis. Los resultados encontrados mostraron variaciones en los niveles de glucosa, sin embargo no tiende a normalizar la concentración de la glucosa. Estos resultados, indican por que posiblemente este efecto este condicionado por los diferentes flavonoides presentes en la estructura de Citrus aurantium (naringina, hesperidina, nobiletina). Esta última premisa, puede ser avalada por estudios los cuales señalan que la naringina puede mantene el estado hiperglicemico en ratas diabéticas.

Por otro lado, la administración de Citrus aurantium a los grupos de animales de estudio no mostró diferencias en los niveles de colesterol con respecto a las ratas diabéticas control (sin tratamiento), sin embargo el efecto inducido por Citrus aurantium sobre los triglicéridos mostró incrementos dependientes de la dosis con respecto a los controles. Es importante mencionar que este fenómeno, es contrario a los reportes de Sharma et al. (2008), los cuales indican que la administración de un extracto de Citrus aurantium induce un efecto hipolipídico, sin embargo aquí cabe mencionar que en este reporte, el extracto fue disuelto en una solución alcohólica, lo que podría condicionar sus resultados.

En conclusión, estos datos y los resultados encontrados en este trabajo señalan: i) La Cnidoscolus chayamansa induce un efecto hipoglucemiante dependiente de la dosis y ejerce un efecto indirecto sobre el perfil lipídico; ii) A dosis bajas Ruta graveolens disminuye los niveles de glucosa, sin embargo el incremento en la dosis trae como consecuencia variación en la concentración de glucosa, colesterol y triacilglicéridos; iii) Citrus auriantum disminuye los niveles de glucosa, sin embargo este efecto no regula la concentración de glucosa en condiciones normales e incrementa los niveles de triglicéridos y peso corporal en una manera dosis-dependiente; y iv) Los efectos inducidos por Cnidoscolus chayamansa, Ruta graveolens y Citrus auriantum pueden depender del tipo de flavonoides presentes en la estructura de las plantas.

\section{BIBLIOGRAFÍA}

Agraa SE, Badwi SM, Adam SE 2002. Preliminary observations on experimental Ruta graveolens toxicosis in Nubian goats. Trop Anim Health Pro 34: 271-81.

Allain CC, Poon L, Chan SG, Richmond W, Fu P 1974. Enzymatic determination of total serum cholesterol. Clin Chem 20: 470-475.

Andrade A, Wiedenfeld H 2001. Hypoglycemic effect of Cecropia obtusifolia on streptozotocin diabetic rats. $J$ Ethnopharmacol 78: 145-149.

Bayne K 1996. Revised guide for the care and use of laboratory animals available. Am Physiol Soc Physiol 39: 208-211.

Baynes J 1991. Role of oxidative stress in development of complications in diabetes. Diabetes 40: 405-412.

Buccolo G, David H 1973. Quantitative determination of serum triglycerides by the use of enzymes. Clin Chem 19: 476482.

Cheng Z, Jing C, Li-Na G, Yong C 2008. Influence of dihydromyricetin on the blood glucose concentration and early kidney's damage in impaired glucose tolerance rats. Jingxi Hua Gong 25: 966-969.

Colker C, Kalman D, Torina G, Perlis T 1999. Effects of Citrus aurantium extract, caffeine, and St. John's Wort on body fat loss, lipid levels, and mood states in overweight healthy adults. Curr Ther Res Clin E 60: 121-153.

Coronado G, Thompson B, Tejeda S, Godina R 2004. Attitudes and beliefs among mexican americans about type 2 diabetes. J Health Care Poor U 15: 576-588.

Dyer A, Stamler J, Berkson O, Lepper M, McKean H 1977. Alcohol consumption, cardiovascular risk factors, and mortality in two Chicago epidemiologic studies. Circulation 56: 1067-1074.

Ezzati M, Henley J, Thun M, Lopez A 2005. Ole of smoking in global and regional cardiovascular mortality. Circulation 112: 489-497.

Fossati P, Prencipe L 1982. Serum triglycerides determined colorimetrically with an enzyme that produces hydrogen peroxide. Clin Chem 28: 2077-2080.

Fugh-Berman A, Myers A 2004. Citrus aurantium, an ingredient of dietary supplements marketed for weight loss: current status of clinical and basic research. Exp Biol Med 229: 698-704.

Funke I, Melzig M 2006. Traditionally used plants in diabetes therapy-phytotherapeutics as inhibitors of a-amylase activity. Rev Bras Farmacogn 16: 1-5.

Gaziano AT 2005. Cardiovascular disease in the developing world and its cost-effective management. Circulation 112: 3547-3553.

Gonzales R, Flores M, Quintero M, Karchesy J 2003. Flavonoid and cyanogenic contents of Chaya (Spinach Tree). Plant Food Hum Nutr 58: 1-8.

Gordon D, Probstfield J, Garrison R, Neaton J, Castelli W, Knoke J 1989. High-density lipoprotein cholesterol and cardiovascular disease. Four prospective american studies. Circulation 79: 8-15.

Harris MI, Flegal KM, Cowie CC, Eberhardt MS, Goldstein DE, Little RR 1998. Prevalence of diabetes, impaired fasting glucose, and impaired glucose tolerance in U.S. adults. The third national health and nutrition examination 
survey, 1988-1994. Diabetes Care 21: 518-24.

Hawkins R 2005. Evaluation of roche accu-chek go and medisense optium blood glucose meters. Clin Chim Acta 353: 127-135.

Hawthorne S, Grabanski C, Martin E, Miller D 2000. Comparisons of Soxhlet extraction, pressurized liquid extraction, supercritical fluid extraction and subcritical water extraction for environmental solids: recovery, selectivity and effects on sample matrix. J Chromatogr 892: 421-433.

Heimler D, Pieron A, Tattini M, Cimato A 1992. Determination of flavonoids, flavonoid glycosides and biflavonoids in Olea europae L. leavels. Chromatographie 33: 349-373.

Hitchcock P, Pugh J, Larme A, Marsh G 1997. The use of traditional plant medicines for non-insulin dependent diabetes mellitus in south Texas. Phytother Res 11: 512517.

Je H, Shin C, Park S, Yim S, Kum C 2002 . Combination of vitamin $\mathrm{C}$ and rutin on neuropathy and lung damage of diabetes mellitus rats. Arch Pharm Res 25:184-190.

Jong K, Chong K, Kun S 2000. Inhibition of alpha-glucosidase and amylase by luteolin, a flavonoid. Biosci Biotech Bioch 64: 2458-2461.

Knowler WC, Barrett-Connor E, Fowler SE, Hamman RF, Lachin JM 2002. Reduction in the incidence of type 2 diabetes with lifestyle intervention or metformin. New Engl J Med 346: 393-403.

Lott J, Turner K 1975. Evaluation of trinder's glucose oxidase method for measuring glucose in serum and urine. Clin Chem 21: 1754-1760.

Mahabir D, Gulliford M 1997. Use of medicinal plants for diabetes in Trinidad and Tobago. Rev Panam Salud Publica 1: 174-179.

Moller D 2001. New drug targets for type 2 diabetes and the metabolic syndrome. Nature 414: 821-827.

Nuraliev I, Avezov GA 1992. The efficacy of quercetin in alloxan diabetes. Eksp Klin Farmakol 55: 42-44.

Oliveira E, Silva D, Gonçalves S, Danni F 1998. Intensive bloodglucose control with sulphonylureas or insulin compared with conventional treatment and risk of complications in patients with type 2 diabetes (UKPDS 33). UK Prospective Diabetes Study (UKPDS) Group. Lancet 352: 837-53.

O'Sullivan D, Cashman W 1970. Blood glucose variations and clinical experience with glibenclamide in diabetes mellitus. Brit Med J 2: 572-574.

Palos SG del Rosario 2007. Evaluación de la actividad antioxidante de la chaya (Cnidoscolus chayamansa) en un modelo experimental de diabetes en ratas wistar. Tesis Maestria en Tecnología Avanzada No. A50074. cicata-qro p. 1-106.

Penicaud L, Hitier Y, Ferre P, Girard J 1989. Hypoglycaemic effect of metformin in genetically obese (fa/fa) rats results from an increased utilization of blood glucose by intestine. Biochem J 262: 881-885.

Pérez R, Pérez C, Zavala M 1998. Actividad hipoglucemiante de Bouvardia terniflora, Brickellia veronicaefolia y Parmentiera edulis. Salud Publ Mex 40: 354-358.

Rajasekaran S, Sivagnanam K, Subramanian S 2005. Antioxidant effect of Aloe vera gel extract in streptozotocin-induced diabetes in rats. Pharmacol Rep 57: 90-96.

Rates S 2001. Plants as source of drugs. Toxicon 39: 603-613.

Rubin RJ, Altman WM, Mendelson DN 1994. Health care expenditures for people with diabetes mellitus, 1992. J Clin Endocr Metab 78: 809A-809F.

Saieed P, Reza R, Abbas D, Seyyedvali R 2006. Inhibitory effects of Ruta graveolens L. extract on guinea pig liver aldehyde oxidase. Chem Pharm Bull 54: 9-13.

Salpeter S, Greyber E, Pasternak G, Salpeter E 2003. Risk of fatal and nonfatal lactic acidosis with metformin use in type 2 diabetes mellitus. Arch Intern Med 163: 2594-2602.

Santos K, Oliveira T, Nagem T, Pinto A, Oliveira M 1999. hypolipidaemic effects of naringenin, rutin, nicotinic acid and their associations. Pharmacol Res 40: 493-496.

Sharma M, Fernandes J, Ahirwar D, Jain R 2008. Hypoglycemic and hypolipidimic activity of alcoholic extract of Citrus aurantium in normal and alloxan-induced diabetic rats. Pharmacologyonline 3: 161-171.

Siguira M, Ogawa K, Masamichi Y 2006. Effect of chronic administration of fruit extract (Citrus unshiu Marc.) on glucose tolerance in GK rats, a model of type 2 diabetes. Biosci Biotech Bioch 70: 293-295.

Singh P, Kar A 2007. Antidiabetic potential of Citrus sinensis and Punica granatum peel extracts in alloxan treated male mice. Biofactors 31: 17-24.

Srinath R, Yusuf S 1998. Emerging epidemic of cardiovascular disease in developing countries. Circulation 97: 596601.

Srinivasan K, Kaul C, Ramarao P 2005. Partial protective effect of rutin on multiple low dose streptozotocin-induced diabetes in mice. Indian J Pharmacol 37: 327-328.

Stamler J, Vaccaro O, Neaton JD, Wentworth D 1993. Diabetes, other risk factors, and 12-year cardiovascular mortality for men screened in the multiple risk factor intervention trial. Diabetes Care 16: 434-44.

Wongpaitoon V, Mills P, Russell R, Patrick R 1981. Intrahepatic cholestasis and cutaneous bullae associated with glibenclamide therapy. Postgrad Med J 57: 244-246.

Zárate A, Tene C 1999. Nuevos fármacos en el tratamiento de la diabetes mellitus tipo 2. Gac Med Mex 135: 91-93. 